\title{
Self-tuning laser speckle contrast analysis based on multiple exposure times with enhanced temporal resolution
}

D. Zölei

zoleid@titan.physx.u-szeged.hu

\section{T. Smausz}

\section{B. Hopp \\ F. Bari}

\begin{abstract}
Department of Optics and Quantum Electronics, University of Szeged, Dóm tér 9, H-6720 Szeged, Hungary

Department of Medical Physics and Informatics, University of Szeged, Korányi fasor 9., H-6720 Szeged, Hungary

Department of Optics and Quantum Electronics, University of Szeged, Dóm tér 9, H-6720 Szeged, Hungary

MTA-SZTE Research Group on Photoacoustic Spectroscopy, University of Szeged, Dóm tér 9, H-6720 Szeged, Hungary
\end{abstract}

Department of Optics and Quantum Electronics, University of Szeged, Dóm tér 9, H-6720 Szeged, Hungary

Department of Medical Physics and Informatics, University of Szeged, Korányi fasor 9., H-6720 Szeged, Hungary

Laser Speckle Contrast Analysis (LASCA) was introduced in 1981. Since then, several enhancements were applied to it. Nowadays, the technique can provide relatively high accuracy as well as high temporal and spatial resolution during the examination of ocular or cerebral tissues. However, in the case of skin, the results are highly affected by the intensive scattering on the skin surface, as the scattering on the non-moving parts of the sample lead to the detrimental decrease of the accuracy. We present a LASCA method based on the use of multiple exposure times, combined with the switching-mode control of the light intensity and a special sampling technique to achieve near to real-time measurement of the skin perfusion. The system based on our method is able to automatically handle the destructive effect of the skin surface and re-tune itself according to the changes of the sample, while it provides full-field perfusion maps with high accuracy, without the need of any precalibrations.

[DOI: http://dx.doi.org/10.2971/jeos.2013.13053]

Keywords: Laser speckle contrast analysis, multiple exposure times, blood perfusion of skin, enhanced temporal resolution, full-field perfusion map

\section{I NTRODUCTION}

The LASCA method for the measurement of the blood flow was introduced by Fercher and Briers in 1981 [1]. If a camera is looking at a moving sample or a sample containing moving parts which is illuminated by coherent light, the interference pattern on the surface of the CCD chip changes in time. The blurring rate of the time-integrated speckle pattern is related to the actual speed of the motion. In the case of living tissues, the blurring is primarily caused by the red blood cells which act as moving scattering centres. This efficient tool is widely used for the mapping of the blood flow and the blood perfusion of cerebral [2] and ocular [3] tissues.

The contrast, an objective property of the pattern, is defined as

$$
K=\frac{\sigma}{\langle I\rangle},
$$

where $\sigma$ is the standard deviation, and $\langle I\rangle$ is the average of the intensities. The local contrast is usually measured on $5 \times 5$ or $7 \times 7$ pixel large sections of the image: by the use of this technique, one can calculate the contrast map of the image $[4,5]$.

Ideally, if the speckle pattern is created by polarized light and the illuminated sample only contains moving scattering parts (e. g. suspension of latex microspheres), the value of the contrast depends on the characteristic speed of the movement and the exposure time:

$$
K^{2}(T)=\frac{1}{T} \int_{0}^{T} C^{2}(t, \tau) d t,
$$

where $T$ is the exposure time, $\tau$ is the autocorrelation time of the intensity fluctuations of the speckles (which is supposed to be inversely proportional to the average velocity), and $C(t, \tau)$ is the autocovariance function of the intensity values of the speckle pattern. If the scatterers are moving with Lorentzian speed distribution, the autocovariance function takes the form of $C=\exp (-T / \tau)$ [1]. However, during real measurements, if the speckle pattern is captured by a digital camera, the size mismatch of the speckles and the pixels, the multiple scattering, the depolarisation and other non-ideal circumstances lead to the decrease of the contrast. These were taken into account by Rojas et al. by the introduction of the $\beta$ normalization factor [6]. Besides, its mathematical background was also enhanced by Bandyopadhyay et al., as they showed that greater weight 
should be given to the faster intensity changes by the use of the $(1-t / T)$ weighting [7]:

$$
K^{2}(T)=\beta^{2} \frac{1}{T} \int_{0}^{T}\left(1-\frac{t}{T}\right) \cdot C^{2}(t, \tau) d t,
$$

where $\beta=\lim _{T \rightarrow 0} K(T)$. Since the value of $\beta$ depends on the actual setup, it has to be determined for each individual experimental arrangement.

However, these models do not take into account the light which is scattered from the non-moving parts of the sample (static scattering) despite of its considerable detrimental effect on the accuracy and reliability of the results in the case of skin perfusion measurements. Parthasarathy et al. [8] and Zakharov et al. [9] proposed a method for calculating the contribution of the static scattering.

In an earlier work we showed that the static scattering can be handled in a relatively simple way by the combination of the use of multiple exposure times and the introduction of two correctional parameters [10]. Assuming that

$$
K^{2}=\frac{\sigma_{\text {dynamic }}^{2}+\sigma_{\text {static }}^{2}}{\langle I\rangle^{2}},
$$

where $\sigma_{\text {dynamic }}$ is the standard deviation of the pattern created by the moving elements, and $\sigma_{\text {static }}$ is the standard deviation of the pattern created by the static elements, one can obtain that the contrast values only depend partially on the exposure time. Assigning the weight of $P_{1}$ to the dynamic part (which actually depends on the exposure time) and $P_{2}$ to the static part one finds that

$$
K^{2}(T)=\frac{1}{T} \int_{0}^{T}\left[P_{1}^{2} \cdot\left(1-\frac{t}{\tau}\right) \cdot C^{2}(t, \tau)+P_{2}^{2}\right] d t,
$$

hence $\lim _{T \rightarrow 0} K^{2}(T)=P_{1}^{2}+P_{2}^{2}$ and $\lim _{T \rightarrow \infty} K^{2}(T)=P_{2}^{2}$ [10]. The processing of the integral results in

$$
K^{2}(T)=P_{1}^{2} \frac{\tau^{2}}{2 T^{2}}\left[\exp \left(\frac{-2 T}{\tau}\right)-1+\frac{2 T}{\tau}\right]+P_{2}^{2} .
$$

The usefulness of our method was demonstrated in [10] for synthetic sample and in [11] for skin. We showed that no precalibration of the system was needed to achieve an increased reliability. In the case of cerebral blood flow monitoring, the contribution of static scattering can be neglected, therefore the use of a well-chosen single exposure time combined with the predetermination of $\beta$ might also provide acceptable accuracy [12].

Although our method based on the use of multiple exposure times provided an increased accuracy, its temporal resolution was much lower than that of the conventional single-exposure ones. The problem can be explained as follows. Since the local contrast values are usually measured on $5 \times 5$ or $7 \times 7$ pixel areas and the speckles have the size similar to that of the pixels, the local contrast values are highly influenced by the random nature of the interference pattern, thus the local contrast values are affected by a small (generally around or less than 4) signalto-noise ratio (SNR) [13]. The spatial averaging of the contrast values would result in the decrease of the lateral resolution which is unacceptable in many cases. This can be avoided by the temporal averaging of the (spatial) local contrast values. However, in the case of multiple exposure times, this has to be performed for each exposure time separately, which dramatically decreases the temporal resolution of the measurements.

Here we demonstrate a near to real-time multi-exposure method for the measurement of the skin perfusion, which is based on the combination of a fast and low-cost intensity modulation system and an optimized image sampling and processing algorithm.

\section{EXPERIMENTAL SETUP}

The experimental setup is shown in Figure 1. The sample (skin of the forearm) was illuminated by the polarized light of a Sanyo DL-8141-002 laser diode (808 nm wavelength, $200 \mathrm{~mW}$ maximum output power). Its current was supplied by a Thorlabs LDC 220C driver which was externally controlled by a home-made programmable signal generator. The size of the area of the illuminated tissue was $25 \mathrm{~cm}^{2}$. The sample was imaged by a PixeLINK PL-B741F camera (monochrome, CMOS, $1280 \times 1024$ maximum resolution, 10 bits depth, 1536:1 signalto-noise ratio). The objective was equipped with a colour filter to reduce the effect of the background illumination and a polarizer to exclude the light which was directly reflected from the surface.

The camera was working with a constant integration time of $100 \mathrm{~ms}$ and the actual exposure time of the speckle images was controlled in the 1-100 ms range by the illumination system. Since the operating wavelength of the diode laser was very sensitive to the changes of the drive current and the temperature, a current modulation method was elaborated. This assured the mode-hop free operation of the laser diode while

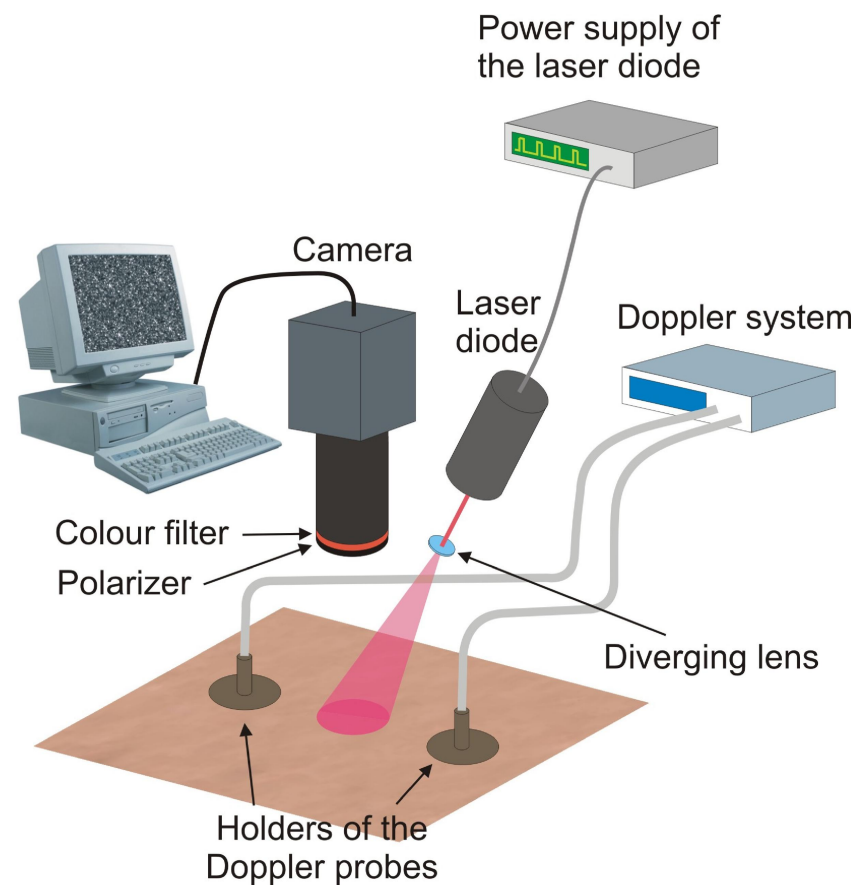

FIG. 1 The experimental setup used for the perfusion measurements. 
maintaining the same average grey scale level for each exposure time value. The basic idea is that each exposure is divided into the same number of short similar flashes, generated by the modulation of the laser diode current with appropriate square voltage signals [14]. Each exposure consisted of 10 flashes. The length of each flash was $47 \mu$ s and the peak value of the driving current during them was $200 \mathrm{~mA}$. The exposure time - corresponding to the full length of the flash train - was controlled by the separation time between the individual flashes. The applied exposure times were 1, 2, 5, 10, 20,50 , and $100 \mathrm{~ms}$, respectively, and the images were captured with a frame rate of $5 \mathrm{fps}$. Background images were taken with the laser light switched off to eliminate the effect of the ambient light. The fixed integration time of the camera highly reduced the spatial noise and random changes, which were caused by the non-linear behaviour of the camera during the application of variable shutter speeds. These non-linear properties appeared because the flat-field correction (correction of fixed pattern noise) of the camera was valid only for a narrow range of shutter speeds (selected by the user), while a wide range of shutter speeds was apllied.

Though the exposure time could be varied from image to image, the record of a whole series of images could take near to two seconds, which had to be repeated several times in order to decrease the statistical noise. To enhance the temporal resolution and the reaction of the system, we created a sampling technique, which combined the conventional single-exposure and our multi-exposure method. First, a multi-exposure measurement was performed to initialize the $P_{1}$ and $P_{2}$ parameters on the basis of Eq. (6). After that, the images were recorded in the following repeating sequence given in ms units: ... 010-1-10-2-10-5-10-20-10-50-10-100-10... The 10 ms was chosen as the monitoring exposure time for the determination of the inverse correlation time considering the $P_{1}$ and $P_{2}$ as known parameters. Obviously, the $0 \mathrm{~ms}$ exposure time corresponded to the dark reference image. The images recorded with exposure times different from $10 \mathrm{~ms}$ were used for the refreshing of the $P_{1}$ and $P_{2}$ parameters as they might have been influenced by actual perfusion state of the tissue (as shown in [11]). This means that this method made the system able to re-tune itself automatically according to the light originating from the environment and the changes of the scattering properties of the sample, while it could provide the actual perfusion rate of the sample with a rate of $2.5 \mathrm{~Hz}$.

The simplest way for eliminating the effect of the statistical noise of the contrast maps and smoothing the signal was the application of running average:

$$
\begin{gathered}
\sigma_{\text {avg }}^{2}=(m-1) \cdot \sigma_{\text {old }}^{2}+m \cdot \sigma_{\text {new }}^{2}, \\
\langle I\rangle_{\text {avg }}=(m-1) \cdot\langle I\rangle_{\text {old }}+m \cdot\langle I\rangle_{\text {new }}, \\
K_{\text {avg }}^{2}=\frac{\sigma_{\text {avg }}^{2}}{\langle I\rangle_{\text {avg }}^{2}}, \\
\tau_{\text {avg }}^{-1}=(n-1) \cdot \tau_{\text {old }}^{-1}+n \cdot \tau_{\text {new }}^{-1},
\end{gathered}
$$

where the avg, old, new subscripts stand for the current average, the old average and the value based on the last image, respectively. We found that the SNR of the averaged results and the reaction time of the system could be optimized by the separate averaging of the contrast and the inverse correlation time. The total reaction time of the speckle system was measured by the radical change of the background illumination: we simply turned on a reading lamp during a measurement, which had a light intensity seen by the camera nearly equal to the shiniest speckle peaks of the images. We found that the total reaction time of the system was less than 1 second without the running average $(m=n=1)$, and 2 to 10 seconds with the running average described above. To find the optimal values of $m$ and $n$, we had to find the combination which resulted in the largest relative increase of the SNR compared to the relative increase of the total reaction time. We found that the optimal values for $m$ and $n$ were 0.7 and 0.35 , respectively, in the case of skin.

A two-channel Periflux 4000 contact laser Doppler system was used as reference (Figure 1.) just as described in [11], operating at the wavelength of $635 \mathrm{~nm}$. This Doppler system could provide a sampling rate of $64 \mathrm{~Hz}$ for both channels, and provided the flow rate in perfusion units. The laser Doppler perfusion unit is proportional to the product of the density and the speed of the moving red blood cells and it is supposed to be proportional to the inverse correlation time $(1 / \tau)$. A major drawback of the Periflux 4000 system was that it could only measure the perfusion within a volume of approximately $1 \mathrm{~mm}^{3}$ for each channel. Although the wavelength used in LASCA and Doppler measurements were different, a study of Abbot et al. [15] indicated that results of Doppler imaging using red and infrared light, respectively, are similar on normal (healthy) skin of dorsal hand, if there are no larger vessels under the skin that could influence the results given by the deeper penetrating near-infrared light. Unfortunately, the same area could not be monitored by the Doppler and the speckle system simultaneously. The probes were placed near to each other (with a separation around $5 \mathrm{~cm}$ ) carefully as not to cover any visible blood vessels. Their positions were selected around the area examined by the speckle system, which also did not contain any visible vessels. We used the Doppler system as reference because (theoretically) it could provide the exact value of the perfusion within the volume examined, and the temporal statistics of the Doppler signal was supposed to be very similar to the spatial statistics of the interference pattern seen by speckle system, as shown by Thompson et al. [16].

\section{MEASUREMENTS AND DISCUSSION}

The measurements were performed on the forearm skin of eight healthy volunteers (both men and women, ages between 23 and 50 years, smoking habits not taken into consideration). Two measurements were performed on each volunteer, the length of each measurement was less than 3 minutes, the separation time between them was 25 minutes. The cuff of a sphygmomanometer was put on the arm of each volunteer to create ischemia. The measurements consisted of three parts: 
1. The baseline was recorded before the application of any stimuli.

2. Ischemia was applied on the arm by setting the pressure in the cuff of the sphygmomanometer $30 \mathrm{mmHg}$ above the systolic pressure.

3. Reperfusion occurred after the cuff was released.

The $1 / \tau$ values determined by the speckle system are referred as SCPU (speckle contrast perfusion unit), while the results of the Doppler system are referred ad LDPU (Laser Doppler perfusion unit). Since the actual SCPU and LDPU values vary from person to person, we normalized them with respect to the average baseline value for each measurement individually.

Figure 2 shows the results of a representative measurement. It clearly shows that - aside from the movement artefacts caused by the increased tremor during the ischemia - the results of our speckle and the Doppler system are in good agreement. As the figure shows, the movement artefacts were not seen by the Doppler system because the probes were taped on the skin surface. However, the full-field systems are generally sensitive on the movement of the sample. As Figure 2 shows, the speckle system could follow the changes of the tissue just as fast as the Doppler system. It is remarkable that it could provide this accuracy at a 25 times slower sampling rate compared to the Doppler system (2.5 vs. $64 \mathrm{~Hz}$, respectively). The system was programmed to be able to monitor 4 selected areas simultaneously. Each plot shows the average SCPU values of the corresponding selected area (sizes of around $1 \mathrm{~cm}^{2}$ ). The square-like areas were selected that they did not overlap each other. They were also distant enough from the tapes of the Doppler-probes which made sure that the accidental movements of the skin would not move the tape into any of the four selected areas. No other special steps were taken when we selected the areas. The calculation of the average SCPU of each area was based on the spatial application of the (8-11) formulas.

Figure 3 shows the summary of the measurements. The nearto real-time measurement made us able to select the time ranges of the measurements which did not contain movement artefacts. The relative LSPU values (as compared to the baseline) were plotted with respect to the related relative LDPU values. Compared to [11], a significant increase of the accuracy can be seen, with a correlation factor of 0.986 . The line fitting was performed that the line crosses the $(100 \%, 100 \%)$ point, which is the baseline for each measurement. The remarkable spread of the measured data can be attributed to the experimental setup similarly to $[11,17,18]$, i. e. the probes of the Doppler system could only be mounted on the skin separately around the area monitored by the speckle system and the Doppler system was able to measure the perfusion within only $\sim 1 \mathrm{~mm}^{3}$ volume per probe. Although the probes were carefully placed to areas where no visible vessels were present; however, the results of some measurements were unusable because - despite the careful preparation - the Doppler probes were put above larger vessels which were invisible for the eye. These results were excluded because they showed a different change of the flow rate than that of the perfusion.

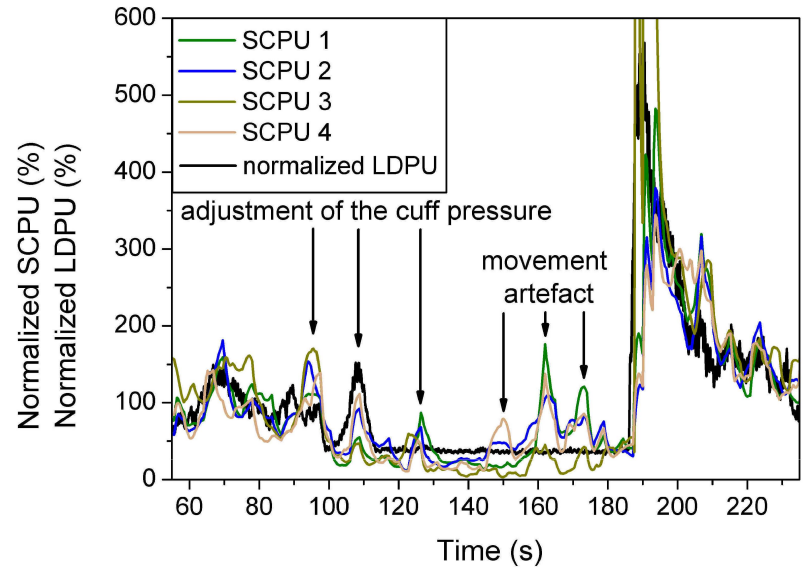

FIC. 2 The relative SCPU and LDPU values (with respect to the average baseline value) during a representative measurement. Though the speckle system was sensitive to the intensive tremor during the ischemia, the results of the two systems are in good agreement. The speckle system was programmed to be able to monitor 4 selected areas simultaneously. Each plot shows the average SCPU values of the corresponding area (size of around $1 \mathrm{~cm}^{2}$ ).

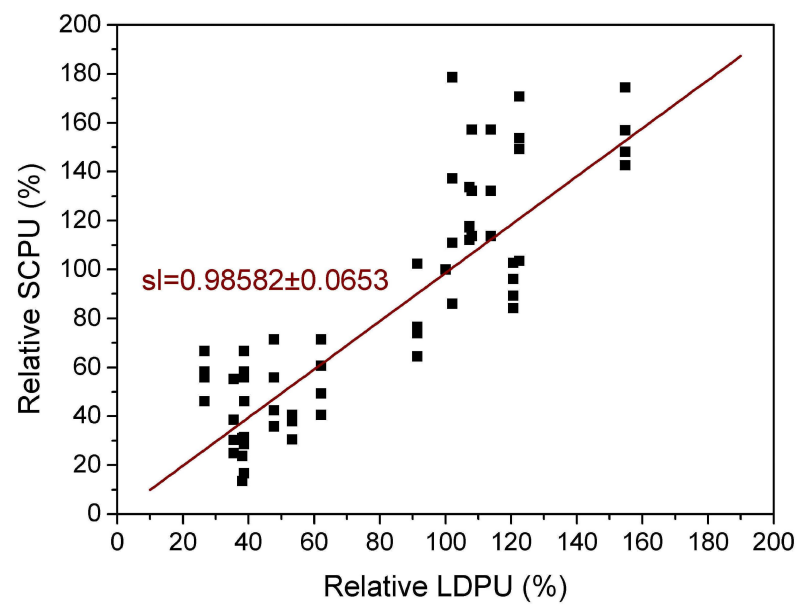

FIC. 3 Correlation between the SCPU and LDPU data. The line fitting was performed as to cross the $(100 \%, 100 \%)$ point, which is the baseline for each measurement

Since the light originating from the laser diode scattered intensively in the skin, the entry and exit points of the individual photons could be as distant as several millimetres from each other. This resulted in the heavy blurring of the scattering information of the undersurface objects, which made the speckle system unable to localize the vessels.

During the measurements, there is a definite need for the insitu, live, full-field monitoring of the perfusion. However, the calculation of a perfusion map with the size of $\sim 300 \times 200$ pixels based on the fitting method described above would take several hours. Therefore we created an advanced method based on lookup tables. Luo et al. also used lookup tables to reduce the calculation time; however, they only applied it to single exposure time measurements, where 1-D lookup tables were needed [19]. The main advantage of our algorithm is the applicability for 7 exposure times, which needed the creation of 7-D lookup tables. As we applied several optimizations, while keeping a low approximation error, one full-field perfu- 


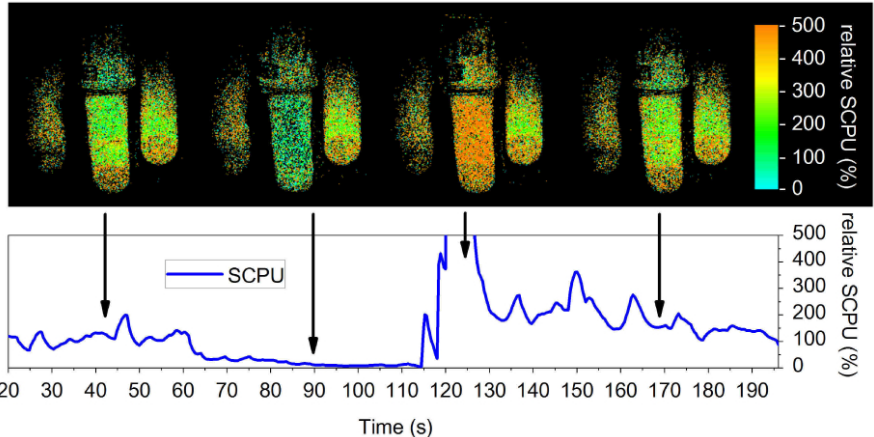

FIG. 4 Slide of perfusion maps cut from a real-time full-field measurement and the average perfusion values of the stimulated finger. The arrows show the corresponding sampling moments of the images. The parts of the measurement are as follows: 20-60 s: baseline, 70-115 s: occlusion, 115-195 s: reperfusion.

sion map could be calculated within less than $200 \mathrm{~ms}$, which allowed the in-situ evaluation during the measurements. Figure 4 shows a slide of perfusion maps (cut from a real-time full-field measurement) and the average perfusion values of the stimulated finger.

\section{CONCLUSIONS}

Multi-exposure LASCA combined with the switching mode control of the laser diode current and our sampling technique can provide as accurate values for the skin perfusion as a Doppler system used in the daily hospital routine. Our system is also able to provide near to real-time full-field results. The technique presented in this article could be an extremely useful tool in the cases of the need of real-time full-field results, like the application of post occlusive reactive hyperaemia (PORH) for the examination of diabetic dermadromes, transplantations, as well as cancerous mutations of the skin.

\section{ACKN OWLEDGEMENT}

The publication is supported by the European Union and co-funded by the European Social Fund through the partial financing of: "Broadening the knowledge base and supporting the long term professional sustainability of the Research University Centre of Excellence at the University of Szeged by ensuring the rising generation of excellent scientists." (Project number: TÁMOP-4.2.2/B-10/1-2010-0012), "Impulse lasers for use in materials science and biophotonics" (TÁMOP-4.2.2.A-11/1/KONV-2012-0060), and the TÁMOP4.2.2.A-11/1/KONV-2012-0035 European Union Grant.

\section{References}

[1] A. F. Fercher, and J. D. Briers, "Flow Visualization by Means of Single-exposure Speckle Photography," Opt. Commun. 37, 326-330 (1981).

[2] A. K. Dunn, A. Devor, H. Bolay, M. L. Andermann, M. A. Moskowitz, A. M. Dale, and D. A. Boas, "Simultaneous imaging of total cerebral hemoglobin concentration, oxygenation, and blood flow during functional activation," Opt. Lett. 28, 28-30 (2003).
[3] M. Nagahara, Y. Tamaki, M. Araie, and H. Fujii, "Real-time blood velocity measurements in human retinal vein using the laser speckle phenomenon," Jpn. J. Ophthalmol. 43, 186-195 (1999).

[4] J. D. Briers, and S. Webster, "Quasi Real-time Digital Version of Single-exposure Speckle Photography for Full-field Monitoring of Velocity or Flow Fields," Opt. Commun. 116, 36-42 (1995).

[5] D. D. Duncan, S. J. Kirkpatrick, and R. K. Wang, "Statistics of Local Speckle Contrast," J. 0pt. Soc. Am. A 25, 9-15 (2008).

[6] L. F. Rojas, D. Lacoste, R. Lenke, P. Schurtenberger, and F. Scheffold, "Depolarization of Backscattered Linearly Polarized Light," J. 0pt. Soc. Am. A 21, 1799-1804 (2004).

[7] R. Bandyopadhyay, A. S. Gittings, S. S. Suh, P. K. Dixon, and D. J. Durian, "Speckle-visibility Spectroscopy: a Tool to Study Timevarying Dynamics," Rev. Sci. Instrum. 76, 093110 (2005).

[8] A. B. Parthasarathy, W. J. Tom, A. Gopal, X. Zhang and A. K. Dunn, "Robust Flow Measurement with Multi-exposure Speckle Imaging," Opt. Express 16, 1975-1989 (2008).

[9] P. Zakharov, A. C. Völker, M. T. Wyss, F. Haiss, N. Calcinaghi, C. Zunzunegui, A. Buck, F. Scheffold, and B. Weber, "Dynamic laser speckle imaging of cerebral blood flow," Opt. Express 17, 13904-13917 (2009).

[10] T. Smausz, D. Zölei, and B. Hopp, “Real Correlation Time Measurement in Laser Speckle Contrast Analysis Using Wide Exposure Time Range Images," Appl. Opt. 48, 1425-1429 (2009).

[11] D. Zölei, T. Smausz, B. Hopp, and F. Bari, "Multiple Exposure Time Based Laser Speckle Contrast Analysis: Demonstration of Applicability in Skin Perfusion Measurements," PCA0 1, 28-32 (2012).

[12] F. Domoki, D. Zölei, 0. Oláh, V. Tóth-Szúki, B. Hopp, and T. Smausz, "Evaluation of Laser-speckle Contrast Image Analysis Techniques in the Cortical Microcirculation of Piglets," Microvasc. Res. 83, 311-317 (2012).

[13] A. C. Völker, P. Zakharov, B. Weber, F. Buck, and F. Scheffold, "Laser Speckle Imaging with an Active Noise Reduction Scheme," Opt. Express 13, 9782-9787 (2005).

[14] T. Smausz, D. Zölei, and B. Hopp, “Laser power modulation with wavelength stabilization in multiple exposure laser speckle contrast analysis," Proc. SPIE 8413, 84131J (2012).

[15] N. C. Abbot, W. R. Ferrell, J. C. Lockhart, J. G. Lowe, "Laser Doppler perfusion imaging of skin blood flow using red and near-infrared sources," J. Invest. Dermatol. 107, 882-886 (1996).

[16] 0. B. Thompson, and M. K. Andrews, "Tissue Perfusion Measurements, Multiple-exposure Laser Speckle Analysis Cenerates Laser Doppler-like Spectra," J. Biomed. Opt. 15, 027015 (2010).

[17] C. J. Stewart, R. Frank, K. R. Forrester, J. Tulip, R. Lindsay, and R. C. Bray, "A Comparison of Two Laser-based Methods for Determination of Burn Scar Perfusion: Laser Doppler Versus Laser Speckle Imaging," Burns 31, 744-752 (2005).

[18] M. Roustit, C. Millet, S. Blaise, B. Dufournet, and J. L. Cracowski, "Excellent Reproducibility of Laser Speckle Contrast Imaging to Assess Skin Microvascular Reactivity," Microvasc. Res. 80, 505-511 (2010).

[19] Z. Luo, Z. Yuan, Y. Pan, and C. Du, "Simultaneous Imaging of Cortical Hemodynamics and Blood 0xygenation Change During Cerebral Ischemia Using Dual-wavelength Laser Speckle Contrast Imaging," Opt. Lett. 34, 1480-1482 (2009). 